Cahiers $d u$ MONDE RUSSE

\section{Cahiers du monde russe}

Russie - Empire russe - Union soviétique et États indépendants

$60 / 4 \mid 2019$

Varia

\title{
Arkadi ZELTSER, Unwelcome Memory. Holocaust Monuments in the Soviet Union
}

\section{Catherine Gousseff}

\section{OpenEdition}

\section{Journals}

Édition électronique

URL : https://journals.openedition.org/monderusse/11528

DOI : $10.4000 /$ monderusse. 11528

ISSN : $1777-5388$

\section{Éditeur}

Éditions de l'EHESS

\section{Édition imprimée}

Date de publication : 1 octobre 2019

Pagination : 855-858

ISBN : 978-2-7132-2797-4

ISSN : $1252-6576$

Référence électronique

Catherine Gousseff, «Arkadi ZELTSER, Unwelcome Memory. Holocaust Monuments in the Soviet Union », Cahiers du monde russe [En ligne], 60/4 | 2019, mis en ligne le 01 octobre 2019, consulté le 02 janvier 2023. URL : http://journals.openedition.org/monderusse/11528 ; DOI : https://doi.org/ 10.4000/monderusse. 11528 
Arkadi ZELTSER

\section{Unwelcome Memory}

Holocaust Monuments in the Soviet Union

Jerusalem : Yad Vashem Publications, 2018, 386 p.

Que nous disent les monuments érigés à la suite de la Seconde Guerre mondiale en URSS sur la manière dont les Juifs soviétiques ont commémoré l'Holocauste ? Quelle fut la contribution de ceux-ci à l'érection des signes matériels de la destruction sur les sites mêmes des meurtres de masse ? Dans quelle mesure l'engagement des collectivités juives dans l'entreprise mémorielle d'édification des monuments nous invite-t-il à reconsidérer la posture des Juifs soviétiques à l'époque du stalinisme tardif et au-delà ? C'est à la réponse à ces questions que se consacre Unwelcome Memory d'Arkadi Zeltser, un ouvrage issu à la fois d'une participation au projet collectif «Untold Stories » conduit par Yad Vashem et de la dette personnelle de l'auteur à cette histoire.

Prenant à revers une opinion commune selon laquelle les Juifs soviétiques seraient restés très passifs face à l'entreprise de mise en mémoire de l'Holocauste en URSS, dans un contexte politique de neutralisation volontaire des victimes civiles de la Seconde Guerre mondiale, Arkadi Zeltser cherche à montrer que l'activité mémorielle, principalement à travers l'érection de monuments commémoratifs, atteste au contraire d'un fort engagement collectif des Juifs dans l'inscription matérielle de la mémoire de la destruction sur le territoire de l'URSS. Sur les 2600 sites de massacres répertoriés à ce jour, 733 ont donné lieu à l'érection d'un monument, un chiffre effectivement impressionnant si on le rapporte au silence supposé du passé de l'Holocauste dans l'histoire soviétique. Ces centaines de monuments, que l'auteur désigne comme juifs, ne signifient pas pour autant qu'y figure une mention explicite à l'Holocauste. Il s'agit en revanche d'entreprises où l'initiative de citoyens juifs est clairement attestée. À partir d'une démarche qui combine approche ethnographique, historique et archéologique - compte tenu du 
caractère parfois très ténu des traces laissées —-, l'auteur présente la vaste enquête menée sur ces mausolées, ce qu'on peut connaître de leur histoire et ce qu'elle dit de la mobilisation des Juifs dans l'entreprise mémorielle. La première grande question qu'aborde Arkadi Zeltser est celle du contexte ou plutôt des contextes politiques qui ont permis, malgré le poids des contraintes, de telles initiatives. Après avoir rappelé qu'aucun décret ni directives n'ont explicitement prohibé l'entreprise mémorielle spécifique de l'Holocauste, l'auteur retrace les différents moments forts de l'édification des monuments qui corroborent une périodisation bien identifiée, autant du point de vue de la politique soviétique face aux Juifs, que de celui de l'évolution de la mémoire officielle de la Seconde Guerre mondiale. Le premier temps fort est celui qui va de la libération des territoires aux années 1947-1948, quand se manifestent une série d'initiatives spontanées qui visent en premier lieu à ré-inhumer les victimes en déplaçant les corps des charniers vers les cimetières pour restaurer leur dignité par la sépulture, démarche propre à susciter des projets de monuments et qui donna lieu en tout cas à d'importantes démonstrations de recueillement collectif. Certaines d'entre elles sont parfois même suivies par les autorités, comme lors du rassemblement sur le site de Babij Jar en septembre 1945 (événement qui ne sera pas pour autant le prélude à l'érection d'un monument, bien au contraire, le « syndrome de Babij Jar », tel que l'évoque l'auteur, désignant le silence imposé sur l'Holocauste durant la période soviétique). Cette première phase, bornée par l'émergence de « la lutte contre le cosmopolitisme », mais aussi par l'impératif de reconstruction et de vision d'avenir (dès 1947, le 9 mai, jour de la Victoire, n'est plus un jour férié), est celle où les monuments contiennent les références les plus brèves et les plus signifiantes du point de vue ethnique, notamment à travers l'usage des langues utilisées (yiddish, hébreu) dans les épitaphes. Dès l'examen de cette première période, Arkadi Zeltser fait part d'une attention forte aux localisations précisant, à l'observation des lieux, le facteur déterminant que fut la posture des autorités locales face aux requêtes de commémoration. Červen', non loin de Minsk, rejeta la demande de monument adressée par un groupe de Juifs, tandis que la capitale de la Biélorussie accédait à une requête similaire. Seules Minsk et Vilnius, parmi les grandes villes de l'URSS, ont avalisé les projets commémoratifs, suggérant qu'en règle générale, le contrôle bureaucratique dans les grands centres urbains constituait un obstacle important aux initiatives. Dans les localités de moindre importance, la décision ressortissait à un comité réduit et pouvait être prise en connaissance des requérants. Un élément ressort, constant dans la période soviétique, en cas de refus des autorités, est la motivation indiquée, à savoir la contestation à considérer les Juifs comme les seules victimes des lieux.

Le deuxième temps fort de l'édification de monuments se situe entre le dégel khroutchévien et la stagnation brejnévienne des années 1970, seule période proportionnellement faste jusqu'à la perestroïka qui correspond, par ailleurs, à un renouveau mémoriel important concernant la guerre. L'auteur inscrit ce moment dans un faisceau d'événements qui ont pu contribuer à la fois à une relative libéralisation du comportement des autorités et au regain de mobilisation des collectivités juives, telles la publication du Journal d'Anne Frank (1960) et celle du poème « Babij Jar » 
de Evtušenko dans la Literaturnaja Gazeta (1961) qui, en suscitant moult remous, esquissa un mouvement d'opinion large en URSS. Dans cette période, certains refus des autorités locales d'accéder aux demandes de commémoration ont pu conduire l'initiateur à s'adresser aux échelons supérieurs, voire au sommet du pouvoir pour obtenir gain de cause, comme ce fut le cas d'un activiste de la région de Brjansk, qui finit par recevoir l'agrément de Brežnev. Certaines négociations ont été engagées. Ainsi, entre un groupe de Rudnja et les autorités de Smolensk qui contestaient le bien-fondé d'un monument juif, mais qui, après l'intervention du Soviet suprême de la RSFSR, acceptèrent que les victimes soient ré-inhumées au cimetière juif du lieu et que soit érigé un monument, mais sans aucune mention ethnique ni de l'Holocauste. Dans le cas d'un autre monument à Medžibož, les échanges entre le groupe de Juifs et les autorités locales, réfractaires à l'idée que figurent en épitaphe les victimes juives, le compromis trouvé fut alors de mentionner en russe que le monument était dédié « aux prisonniers du ghetto ».

Après le conflit arabo-israélien de 1967 et durant la stagnation, les initiatives commémoratives se raréfient considérablement. L'accroissement du contrôle idéologique se traduit, notamment, par l'injonction à effacer certaines épitaphes « ethniques » ou à transformer les signes, comme à faire de l'étoile de David une étoile soviétique à cinq branches. La rétrospective temporelle engage aussi une réflexion sur la distribution spatiale des monuments, où il apparaît que ceux-ci furent beaucoup moins nombreux dans les régions tardivement annexées (à l'exception notable, mais non soulignée par l'auteur, de la Lituanie) que dans les régions soviétisées depuis les années 1920, fait qui pourrait s'expliquer à la fois par la radicalité de l'Holocauste dans les régions les plus occidentales et l'émigration massive des survivants de ces régions vers la Pologne à la fin de la guerre. Outre ce constat, aucune logique régionale ne peut être clairement distinguée, dit l'auteur, prenant à témoin l'exceptionnelle mobilisation commémorative de la région de Mogilev, non seulement à l'échelle de la Biélorussie, mais de l'ensemble des territoires anciennement occupés.

Si la parole de ceux engagés dans l'édification de monuments est difficile à restituer, Arkadi Zeltser cherche à reconstituer ces mobilisations au-delà des documents photographiques, nombreux, qui ponctuent la narration et témoignent de la présence de communautés rassemblées dans le jour du souvenir autour d'un mausolée. L'une des attestations de ces engagements est, nous dit l'auteur, la collecte de fonds pour l'édification de mausolées, dont quelques documents cités montrent l'importance. Cependant, le chapitre consacré aux acteurs juifs de l'activité mémorielle est justement celui qui peine le plus à convaincre. Si l'ensemble de l'ouvrage est construit sur une narration qui mobilise la très riche historiographie consacrée aux Juifs soviétiques pour contextualiser les études de cas, force est de constater que, s'agissant de l'analyse des collectivités, des profils des acteurs, de leurs stratégies, le discours historique généralisant prend largement le pas sur l'examen des cas. Arkadi Zeltser affirme, par exemple, que le succès de l'activité mémorielle juive fut lié, pour partie, à leur intégration dans les échelons supérieurs des structures économiques, sociales et culturelles de la société soviétique, mais les mentions où ces 
acteurs apparaissent concrètement restent très marginales. Omniprésents dans le discours de l'auteur, les activistes restent anonymes dans les faits présentés, souvent peu questionnés. Ainsi, s'agissant d'une collecte organisée pour l'édification d'un mausolée à Červen' dans les années 1967-1969, la liste mentionne une concentration de donateurs provenant de Minsk ou de sa région, mais aussi de 77 participants moscovites. On aimerait ici comprendre comment l'initiative s'est fait connaître, a été diffusée, pour susciter une telle mobilisation. L'ambition de l'auteur à vouloir réincarner l'histoire en arguant du fort engagement des communautés juives laisse le lecteur sur sa faim. Le constat renvoie à la question des sources, difficile à appréhender, puisque la majorité des références concernent les copies d'archives soviétiques rassemblées à Yad Vashem et mentionnées seulement par leurs cotes.

\section{Catherine Gousseff} CERCEC, EHESS 